\title{
ZT-SUBGROUPS OF SHARPLY 3-TRANSITIVE GROUPS
}

\author{
by HEINRICH WEFELSCHEID \\ (Received 15th September 1978)
}

A permutation group $G$ operating on a set $M$ is called a ZT-group (Zassenhaus transitive group) if $G$ has the properties (i) and (ii):

(i) $G$ operates 2-transitively on $M$;

(ii) $G_{a, b} \neq\{$ id $\}$ and $G_{a, b, c}=\{$ id $\}$ for distinct elements $a, b, c \in M$.

Here $G_{a, b}=\{\alpha \in G \mid \alpha(a)=a$ and $\alpha(b)=b\}$ denotes the stabilizer of $\{a, b\}$, and $G_{a, b, c}$ the stabilizer of $\{a, b, c\}$, respectively.

In this paper we are looking for all ZT-groups which are subgroups of sharply 3-transitive groups. It is shown that such ZT-groups can be uniquely described by means of certain subgroups $B$ of the multiplicative group $\left(F^{*}, \cdot\right)$ of the KT-field $(F,+, \cdot, \sigma)$ which characterizes the underlying sharply 3-transitive group.

In $\$ 1$ the basic notions and properties of sharply 3-transitive groups are given.

In $\$ 2$ the above mentioned ZT-groups are described.

In $\$ 3$ a method of constructing sharply 3-transitive groups and their ZT-subgroups is treated. It is shown that the smallest ZT-subgroups of these examples are all isomorphic to $\operatorname{PSL}(2, K)$ even if the underlying sharply 3-transitive group is not isomorphic to $\operatorname{PGL}(2, K)$. In the finite case this was already known by Zassenhaus (6), where he determined all finite sharply 3-transitive groups and their ZT-subgroups.

\section{Basic notions and relations}

Definition 1.1. A set $F$ with two binary operations $(+)$ and $(\cdot)$ is called a neardomain ("Fastbereich") if the following axioms are valid:

Fb $1 \quad(F,+)$ is a loop (with neutral element 0 )

Fb $2 \quad a+b=0 \Rightarrow b+a=0$

Fb $3 \quad\left(F^{*}, \cdot\right)$ is a group (with neutral element $1 ; F^{*}:=F \backslash\{0\}$ ).

$\mathrm{Fb} 4 \quad 0 \cdot a=0$, for every $a \in F$.

Fb $5 \quad a \cdot(b+c)=a b+a c$ for all $a, b, c \in F$.

Fb 6 For every pair of elements $a, b \in F$ there exists an element $d_{a, b} \in F^{*}$, such that

$$
a+(b+x)=(a+b)+d_{a, b} \cdot x
$$

for every $x \in F$.

Remark. Each sharply 2-transitive permutation group can be written as the group of linear transformations $x \rightarrow a+m x, m \neq 0$, of a neardomain $(F,+, \cdot)$. (Karzel (2)). 
Definition 1.2. $(F,+, \cdot \sigma)$ is called a $K T$-field, if the axioms KT 1 and $\mathrm{KT} 2$ are valid: KT 1

KT 2

$(F,+, \cdot)$ is a neardomain.

$\sigma$ is an involutory automorphism of the multiplicative group $\left(F^{*}, \cdot\right)$ which satisfies the functional equation:

$$
\sigma(1+\sigma(x))=1-\sigma(1+x) \quad \text { for all } x \in F \backslash\{0,1\}
$$

As one easily can verify the transformations $\alpha, \beta: \bar{F}:=F \cup\{\infty\} \rightarrow \vec{F}$, where $\infty$ denotes an element not in $F$ and

$$
\begin{array}{ll}
\alpha:\left\{\begin{aligned}
\bar{F} & \rightarrow \bar{F} \\
x & \rightarrow a+m x, \\
\infty & \rightarrow \infty
\end{aligned}\right. & a \in F, m \in F^{*} \\
\beta:\left\{\begin{array}{rl}
\bar{F} & \rightarrow \bar{F} \\
x & \rightarrow a+\sigma(b+m x), \\
\infty & \rightarrow a \\
-m^{-1} b & \rightarrow \infty
\end{array} \quad a, b \in F, m \in F^{*}\right.
\end{array}
$$

form a group $T_{3}(\bar{F})$ which operates sharply 3-transitively on $\bar{F}$. Conversely each sharply 3-transitive group is isomorphic as a permutation group to the group $T_{3}(\bar{F})$ of a uniquely determined KT-field (see (4)).

Therefore in the following we will consider each sharply 3-transitive group as being represented in the form $T_{3}(\bar{F})$.

\section{ZT-subgroups of $T_{3}(\bar{F})$}

The main result will be the following theorem:

Theorem 2.1. Let $(F,+, \cdot, \sigma)$ be a $K T$-field. If $B$ is a subgroup of $\left(F^{*}, \cdot\right)$ such that $R \subseteq B, \quad D \subseteq B$ and $\sigma(B) \subseteq B$, where $R:=\left\{a \sigma\left(a^{-1}\right) \in F^{*} \mid a \in F^{*}\right\}$ and $D=$ $\left\{d_{a, b} \in F^{*} \mid a, b \in F\right\}$ then the transformations of the form:

$$
\begin{array}{ll}
\alpha: \begin{cases}x \rightarrow a+m x, & a \in F, m \in B \\
\infty \rightarrow \infty & \end{cases} \\
\beta:\left\{\begin{aligned}
x \rightarrow a-\sigma(b+m x), & a, b \in F, m \in B \\
-m^{-1} b \rightarrow \infty & \\
\infty & \rightarrow a
\end{aligned}\right.
\end{array}
$$

constitute a subgroup $U$ of $T_{3}(\bar{F})$ which is Zassenhaus transitive.

Conversely, to each $U \leqslant T_{3}(\bar{F})$ which is a $Z T$-group, there exists a subgroup $B \leqslant F^{*}$ with $R \subseteq B, D \subseteq B$ and $\sigma(B) \subseteq B$ such that all elements of $U$ have the form $\alpha$ or $\beta$.

Proof. For the first part of the theorem, let $\alpha_{i}: x \rightarrow a_{i}+m_{i} x$ and $\beta_{i}: x \rightarrow$ $a_{i}-\sigma\left(b_{i}+m_{i} x\right)$ for $i=1,2$ with $a_{i}, b_{i} \in F$ and $m_{i} \in B$. 
Then we have:

$$
\begin{aligned}
& a_{i}^{-1}: x \rightarrow-m_{i}^{-1} a_{i}+m_{i}^{-1} x \\
& \beta_{i}^{-1}: x \rightarrow-m_{i}^{-1} b_{i}-\sigma\left[\sigma\left(-m_{i}^{-1}\right) a_{i}+\sigma\left(m_{i}^{-1}\right) x\right] \\
& \alpha_{1} \alpha_{2}: x \rightarrow\left(a_{1}+m_{1} a_{2}\right)+d_{a_{1}, m_{1} a_{2}} m_{1} m_{2} x \\
& \alpha_{1} \beta_{2}: x \rightarrow\left(a_{1}+m_{1} a_{2}\right) \div \sigma\left[\sigma\left(d_{a_{1}, m_{1} a_{2}}\right) \sigma\left(m_{1}\right) b_{2}+\sigma\left(d_{a_{1}, m_{1} a_{2}}\right) \sigma\left(m_{1}\right) m_{2} x\right] \\
& \beta_{1} \alpha_{2}: x \rightarrow a_{1}-\sigma\left(\left(b_{1}+m_{1} a_{2}\right)+d_{b_{1}, m_{1} a_{2}} m_{1} m_{2} x\right) \\
& \beta_{1} \beta_{2}: x \rightarrow\left[a_{1}-\sigma(t)\right]-\sigma\left[\left(-d t+d t \sigma\left(t^{-1}\right) \sigma\left(d_{b_{1}, m_{1} a_{2}}\right) \sigma\left(m_{1}\right) b_{2}\right)\right.
\end{aligned}
$$

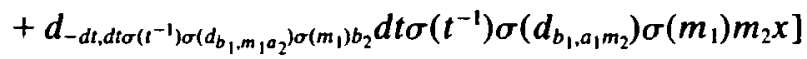

where $t:=b_{1}+m_{1} a_{2} \neq 0$ and $d:=\sigma\left(d_{a_{1}, \sigma(t)}\right)$.

For $t=0$ we get:

$$
\beta_{1} \beta_{2}: \rightarrow\left[a_{1}+\sigma\left(m_{1}\right) b_{2}\right]+d_{a_{1}, \sigma\left(m_{1}\right) b_{2}} \sigma\left(m_{1}\right) m_{2} x .
$$

Because of the properties of $B$ the inverse and the products are all of the form $\alpha$ or $\beta$.

Conversely, let $U$ be a subgroup of $T_{3}(\bar{F})$ which is Zassenhaus-transitive. Since $U_{x, 0}$ is a subgroup consisting of permutations of the form $\alpha: x \rightarrow m x$ the set $A$

$$
A:=\left\{m \in F^{*} \mid m=\alpha(1) \text { with } \alpha \in U_{\infty, 0}\right\}
$$

is a subgroup of $F^{*}$. We have to show that $A$ possesses the required properties.

$U_{\infty}$ consists of transformations of the form $x \rightarrow a+m x$ with $m \in A$. Because of the transitivity of $U_{\infty}$ there exists to each $b \in F$ an $\alpha \in U_{\infty}$ such that $\alpha(0)=b$.

Thus

$$
U_{\infty}=\{x \rightarrow a+m x \mid a \in F \text { and } m \in A\} .
$$

We define now:

$$
H:=\left\{n \in F^{*} \mid \exists a, b \in F \text { such that } \beta \in U, \beta: x \rightarrow a-\sigma(b+n x)\right\} .
$$

Since $U$ is a ZT-group there exists a transformation $\tau \in U$ with $\tau(0)=\infty$ and $\tau(\infty)=0$. Therefore $\tau$ has the form $\tau: x \rightarrow-u \sigma(x)$ where $\tau(1)=u$ and $\sigma(u) \in H$.

Now take some $\beta \in U, \beta(x)=a-\sigma(b+n x)$ and define $\delta(x)=-a+x$. We have $\delta \in U_{\infty}$ and $\tau \delta \beta(x)=u b+u n x$ with $\tau \delta \beta \in U_{\infty}$, i.e. $u n \in A$. Hence $u H \subseteq A$. Also for each $m \in A$ the permutation $x \rightarrow \tau(m x)=-\sigma(\sigma(u) m x)$ lies in $U$ from which $\sigma(u) A \subseteq$ $H$. This implies that $u \sigma(u) A \subseteq u H \subseteq A$ whence $u \sigma(u) \in A$ and so $u H=A$.

Furthermore the above considerations show that for any $n \in H$ and each $a, b \in F$ the permutation $\beta(x)=a-\sigma(b+n x)$ belongs to $U$. The inclusion $D \subseteq A$ follows directly from $\alpha_{1} \alpha_{2}(x)=\left(a_{1}+a_{2}\right)+d_{a_{1}, a_{2}} x$ for $\alpha_{i}(x)=\alpha_{i}+x$ and $i=1,2$.

Now for an arbitrary $\mu \in U_{\infty, 0}$, say $\mu(x)=m x, m \in A$ we get

$$
\tau^{-1} \mu \tau(x)=\sigma\left(u^{-1} m u \sigma(x)\right)=\sigma\left(u^{-1}\right) \sigma(m) \sigma(u) x
$$

whence $\sigma\left(u^{-1}\right) \sigma(m) \sigma(u) \in A$ and so $\sigma(m) \in \sigma(u) A \sigma\left(u^{-1}\right)=u^{-1} A u$, on account of $u \sigma(u) \in A$. Thus $\sigma(A) \subseteq u^{-1} A u$.

Finally we show that $\mu \in A$ : 
For this we consider $\beta(x)=a+\tau(x)$. Then

$$
\begin{aligned}
\tau \beta(x) & =-u \sigma(a-u \sigma(x)) \\
& =-u \sigma(a)-\sigma\left(-\sigma(u) a+\sigma(u) a \sigma\left(a^{-1}\right) \sigma(u) x\right)
\end{aligned}
$$

so that $\sigma(u) a \sigma\left(a^{-1}\right) \sigma(u) \in H=u^{-1} A$ and hence $a \sigma\left(a^{-1}\right) \sigma(u) \in \sigma\left(u^{-1}\right) u^{-1} A$ for each $a \in F^{*}$.

If we put $a=u$ we get $u \in A$ and therefore $A=H$.

Together with $\sigma(A) \subseteq u^{-1} A u$ we get $\sigma(A)=A$. From this follows $a \sigma\left(a^{-1}\right) \in$ $A \sigma(u)^{-1}=A$ for each $a \in F^{*}$, whence $R \subseteq A$.

By computing one gets the

Corollary 2.2. A ZT-subgroup $U$ of $T_{3}(\bar{F})$ is normal if and only if the corresponding subgroup $B$ of $F^{*}$ is normal. In this case $T_{3}(\bar{F}) / U \cong F^{*} / B$.

\section{Examples}

The following theorem of Kerby (12.7 in (3)) shows the way to construct KT-fields. To my knowledge all examples so far known are made in this manner.

Theorem 3.1. Let $(F,+, *)$ be a commutative field and let $A$ be a subgroup of $\left(F^{*}, *\right)$ such that

(i) $Q=\left\{a * a \mid a \in F^{*}\right\} \subseteq A$

(ii) There exists a monomorphism $\pi: F^{*} / A \rightarrow \operatorname{Aut}(F,+, *)$.

(iii) $\tau(x) \in x * A$ for all $x \in F^{*}$ and all $\tau \in \pi\left(F^{*} / A\right)$.

Let $\kappa: F^{*} \rightarrow F^{*} / A$ denote the canonical homomorphism. Then $(F,+, \circ)$

$$
a \circ b=\left\{\begin{array}{lr}
0 & \text { for } a=0 \\
a * a_{\varphi}(b) & \text { with } a_{\varphi}=\pi \kappa(a)
\end{array}\right.
$$

is a (strongly coupled Dickson) nearfield and $(F,+, \circ, \sigma)$ is a $K T$-field with $\sigma(a)=a^{-1}$ (inverse with respect to $(*)$ ).

For instance (see (3), p. 67) take an arbitrary finite or infinite index set $I$. Further let $K$ be a commutative field and $F=K\left(t_{i}\right)_{i \in I}$ the field of rational functions in $|I|$ transcendental indeterminates $t_{i}$ and $\operatorname{grad}_{i} f=\operatorname{grad}_{i}\left(f_{1} / f_{2}\right)=\operatorname{grad}_{i} f_{1}\left(t_{i}\right)-\operatorname{grad}_{i} f_{2}\left(t_{i}\right)$ the degree of the polynomials $f_{1}, f_{2}$ with respect to $t_{i}$.

If we choose $\tau_{i}(k)=k$ for $k \in K, \tau_{i}\left(t_{j}\right)=t_{j}$ for $i \neq j$ and $\tau_{i}\left(t_{i}\right)=1-t_{i}$, then $F^{\varphi}:=(F,+, \circ)$ with

$$
f \circ g:=f \cdot f_{\varphi}(g) \quad \text { where } f_{\varphi}:=\prod_{i \in I} \tau_{i}^{\text {grad }_{i} f}
$$

is a Dickson nearfield and $(F,+, \circ, \sigma)$ with $\sigma(f)=f^{-1}$ (inverse with respect to the multiplication $(\cdot)$ of the commutative field) is a KT-field.

The subgroup $A$ of Th. 3.1 is here

$$
A=\left\{f \in F \mid \operatorname{grad}_{i} f \equiv 0(\bmod 2) \text { for all } i \in I\right\} .
$$


For the rest of the paragraph let $F^{\infty}$ denote the KT-field which is constructed with the help of a commutative field $F$ according to 3.1. Those ZT-subgroups $U \leqslant T_{3}\left(\overline{F^{\Phi}}\right)$ which are at the same time subgroups of PGL $(2, F)$ are characterised by the

Proposition 3.2. Let $F^{\oplus}$ be a KT-field derived according to 3.1 from a commutative field $F$. A ZT-subgroup $U \leqslant T_{3}\left(F^{\varphi}\right)$ is simultaneously a subgroup of $P G L(2, F)$ if and only if the corresponding subgroup $B \leqslant\left(F^{*}, \circ\right)$ satisfies $B \subseteq$ $\operatorname{Ker} \varphi:=\left\{z \in F^{*} \mid z_{\varphi}=\right.$ id $\}$.

Proof. We have to show that the mapping $\Psi$

$$
\psi:\left\{\begin{array}{lll}
U & \rightarrow & \operatorname{PGL}(2, F) \\
\alpha: x \rightarrow a+m \circ x & \rightarrow & x \rightarrow a+m * x \\
\beta: x \rightarrow a-\sigma(b+m \circ x) & \rightarrow & x \rightarrow a-(b+m * x)^{-1}
\end{array}\right.
$$

is a homomorphism. If $B \subseteq \operatorname{Ker} \varphi$ then $m \circ x=m * x$ for all $m \in B$. Denoting the inverse of $a$ with respect to (o) by $a^{-1}$ and with respect to $(*)$ by $a^{-1}$ we get because of $a^{-1}=a_{\varphi}^{-1}\left(a^{-1}\right)$ :

$$
\begin{aligned}
t \circ \sigma\left(t^{-1}\right) & =t * t_{\varphi} \sigma\left(t_{\varphi}^{-1}\left(t^{-1}\right)\right)=t * t_{\varphi}\left[t_{\varphi}^{-1}\left(t^{-1}\right)\right]^{-1} \\
& =t * t_{\varphi} t_{\varphi}^{-1}(t)=t * t .
\end{aligned}
$$

The formulae in the proof of Theorem 2.1 show that $\psi$ is a homomorphism. If, on the other hand, $\psi$ is a homomorphism then $B \subseteq \operatorname{Ker}_{\varphi} . \square$

In all examples furnished by 3.1 the sets $R$ and $Q$ are equal:

$$
R=\left\{a \circ \sigma\left(a^{-1}\right) \mid a \in F^{*}\right\}=\left\{a * a \mid a \in F^{*}\right\}=Q
$$

and $R=Q \subseteq A=\operatorname{Ker}_{\varphi}$. Moreover $Q \leqslant\left(F^{*}, k\right), \quad \sigma(Q) \subseteq Q, Q \leqslant\left(F^{*}, \circ\right)$. So $R=Q$ satisfies the conditions of 2.1 and $B=R$ supplies the smallest ZT-subgroup of $T_{3}\left(\overline{F^{\phi}}\right)$. It is well known that the smallest ZT-subgroup of $\operatorname{PGL}(2, F)$ is $\operatorname{PSL}(2, F)$. Thus 2.1, 3.1 and 3.2 give:

Proposition 3.3. The smallest $Z T$-subgroup $U$ of a sharply 3-transitive group $T_{3}\left(\overline{F^{\varphi}}\right)$ where $F^{\Phi}$ is constructed as in 3.1 , is isomorphic to $\operatorname{PSL}(2, F)$.

Finite KT-fields $F^{\varphi}$ possess only two subgroups $B \leqslant\left(F^{*}, \circ\right)$ relevant to 2.1 namely $F^{*}$ and $R$ if $|F|$ is odd and only one such subgroup namely $F^{*}$ if $|F|$ is even (6).

In order to get examples of ZT-groups which are not simultaneously ZT-subgroups of $\operatorname{PGL}(2, F)$ we have to look for subgroups $B \leqslant\left(F^{*}, \circ\right)$ which satisfy $\sigma(B)=B$, $R \subseteq B$ but $B \subset \operatorname{Ker} \varphi$.

We mention here only two possibilities:

I. Let $(F,+, \cdot)$ be a commutative field, $A \leqslant F^{*}$ and $\tau \in \operatorname{Aut}(F,+, \cdot)$ such that: $\tau^{2}=\mathrm{id}, \tau \neq \mathrm{id}, \tau(A)=A$ and $\left[F^{*}: A\right]=2$. Then $(F,+, \circ, \sigma)$ is a KT-field where $\sigma(a)=a^{-1}$ and

$$
a \circ b=\left\{\begin{array}{ll}
a \cdot b & \text { if } a \in A \\
a \cdot \tau(b) & \text { if } a \notin A^{.}
\end{array} \quad\right. \text { (see (4), p. 232). }
$$


If we choose $\tau$ such that the fixed point field $F_{\tau} \not \subset A \cup\{0\}$ there is a $t \in F_{r} \backslash A$. We define $B=Q \cup Q t$. The set $B$ is a group with $Q=R \subseteq B$ and $\sigma(B) \subseteq B$ but $B C A=$ $\operatorname{Ker}_{\varphi}$.

II. Let $F$ be a KT-field constructed according to 3.1 such that $A=\operatorname{Ker} \varphi$ is a commutative subgroup of index $\left[F^{*}: A\right]=2^{n}, n \geqslant 2$. (More details of this construction can be found in Kerby ( $3 ;$ p. 67).) Then one can easily find a set $B$ with the properties:

$$
\begin{aligned}
& (B, \circ) \leqslant\left(F^{*}, \circ\right) \\
& (B, \cdot) \leqslant\left(F^{*}, \cdot\right) \\
& A \subseteq B \\
& {\left[F^{*}: B\right]=2^{m}<2^{n}=\left[F^{*}: A\right] .}
\end{aligned}
$$

Thus $B \subset \operatorname{Ker} \varphi, \sigma(B)=B, R \subseteq B$ and $B$ satisfies the conditions of 2.1.

For instance let $|I| \geqslant 2$ be as in the example following Theorem 3.1 and take for $B$ :

$$
B=\left\{f \in K\left(t_{i}\right)_{i \in I} \mid \operatorname{grad}_{j} f \equiv 0(\bmod 2) \text { with } j \in J\right\},
$$

where $J$ is a proper subset of $I$.

\section{REFERENCES}

(1) D. Gorenstein, Finite Groups (Harper \& Row, New York, 1968).

(2) H. KARZEL, Zusammenhänge zwischen Fastbereichen, scharf zweifach transitiven Permutationsgruppen und 2-Strukturen mit Rechtecksaxiom, Abh. Math. Sem. Univ. Hamburg, 32 (1968), 191-206.

(3) W. KERBY, Infinite sharply multiple transitive groups (Hamburger Mathematische Einzelschriften, Neue Folge Heft 6, Göttingen 1974, Vandenhoek und Ruprecht).

(4) W. KERBY and H. WEFELSCHEID, Über eine scharf 3-fach transitiven Gruppen zugeordnete algebraische Struktur, Abh. Math. Sem. Hamburg 37 (1972), 225-235.

(5) W. KERBY and H. WEFELSCHEID, Über eine Klasse von scharf 3-fach transitiven Gruppen, J. f. reine und angew. Mathematik, 268/269 (1974), 17-26.

(6) H. ZASSENHAUS, Kennzeichnung endlicher linearer Gruppen als Permutationsgruppen, Abh. Math. Sem. Hamb. Univ. 11 (1934), 17-40.

FACHBEREICH MATHEMATIK

DER UNIVERSITÄT DUISBURG

LOTHARSTR. 65

4100 DuisBurg 1 